\title{
SUR LE DOSAGE DE L'ANTIPYRINE DANS LE PLASMA SANGUIN
}

\author{
PAR \\ B.-L. DUMONT $\left(^{1}\right)$ \\ Station de Recherches sur l'Élevage, C.N.R.Z., Jouy-en-Josas.
}

L'utilisation de l'antipyrine dans la mesure in vivo de l'eau totale de l'organisme a été introduite, chez l'homme, par SoBERMAN et BroDIE (I), qui proposèrent également une méthode de dosage de cette substance dans le plasma sanguin et les différents tissus (2).

Depuis, la détermination de la masse hydrique corporelle a donné lieu à de nombreux travaux utilisant cette méthode de dosage.

$A u$ cours d'une étude de la distribution de l'antipyrine chez le porc et le mouton (3-4), nous avons été amenés à utiliser la méthode de BroDIE.

Nos différents essais nous ont conduits à préciser les divers points de détail de la méthode originale et à y apporter un certain nombre de modifications, relatives au dosage de l'antipyrine dans le plasma sanguin.

\section{I. - La méthode de Brodie}

Elle est basée sur le dosage, à $350 \mathrm{~m}$ milieu sulfurique.

Le détail des opérations peut être résumé ainsi (fig. I) :

$I^{0}$ Prendre comme blanc l'eau sulfurique $\left(3 \mathrm{~cm}^{3}\right.$ eau bidistillée + I goutte de $\left.\mathrm{SO}_{4} \mathrm{H}_{2} 4 \mathrm{~N}\right)$;

Ajouter aux différentes cuves contenant les solutions à analyser $\left({ }^{2}\right)$ I goutte de $\mathrm{SO}_{4} \mathrm{H}_{2} 4 \mathrm{~N}$;

Faire le o de densité optique sur l'eau sulfurique ;

I ire la densité optique des différentes cuves ;

Soit D la densité de l'une de ces cures.

$2^{\circ}$ Ajouter ensuite à chacune des cuves (y compris le blanc sulfurique), 2 gouttes de nitrite de soude à 0,2 p. Ioo ;

Après 20 minutes, lire la densité optique de chaque cuve en faisant le o de l'appareil sur la première cuve : eau sulfurique + nitrite ; laire.

(1) Ce travail a été réalisé avec la collaboration technique de ylle Éléonora Hudzik.

(2) Soit des solutions d'antipyrine, soit des plasmas déféqués, soit des résidus d'extraction tissu- 

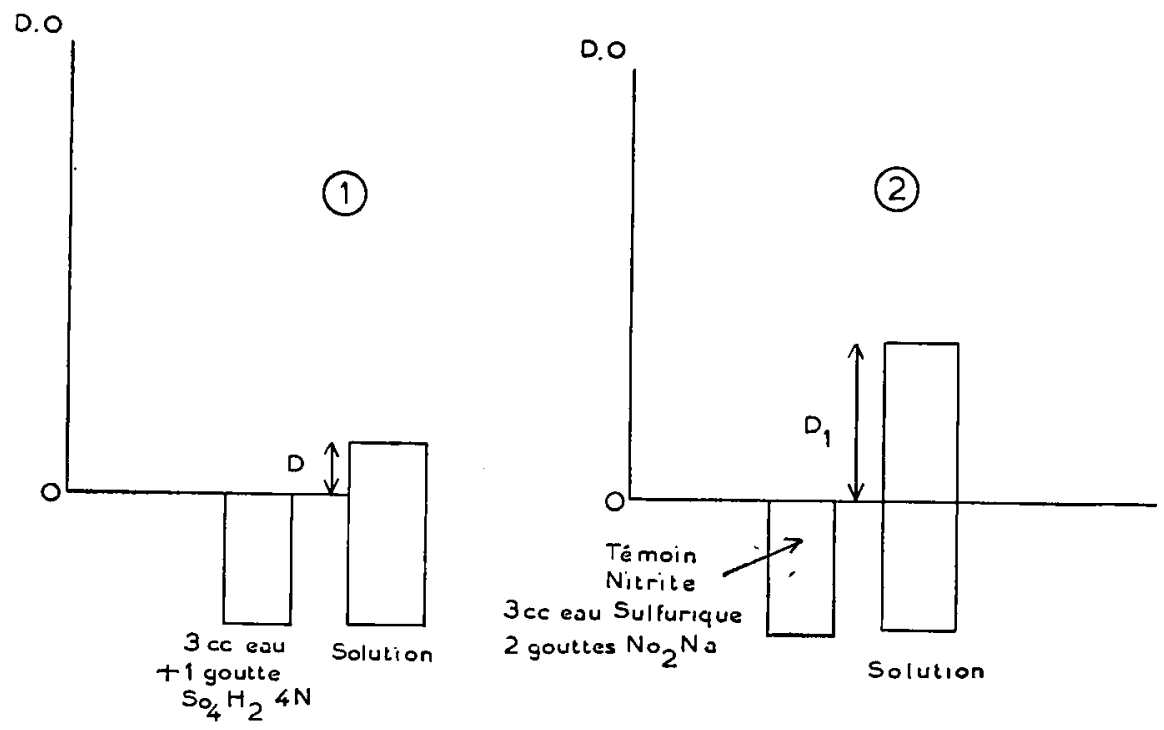

Fig. I. - Schena de la méthode de Brodie.

Soit $\mathrm{D}_{1}$ la densité optique de la cuve considérée en $\mathrm{I}^{0}$; Soustraire $\mathrm{D}$ de $\mathrm{D}_{1}$ pour avoir 1'absorption de la solution.

\section{II. - Critique de la méthode}

\section{a) Rôle du témoin nitrite}

Le fait de soustraire $D$ de $D_{1}$ pour obtenir l'absorption de la solution considérée est expliqué ainsi par BronIE : la faible densité optique de la solution avant l'addition de nitrite n'est pas changée par le nitrite et doit donc être prise en considération, en soustrayant sa valeur (c'est-àdire $D)$ de la valeur finale $D_{1}$.

On doit dissocier, à ce sujet, le cas des solutions pures d'antipyrine et le cas des plasmas.

\section{$\mathrm{I}^{\circ}$ Cas des solutions d'antipyrine}

La densité optique de ces solutions par rapport à l'eau sulfurique est généralement faible, de l'ordre de quelques millièmes de densité, même pour des solutions assez concentrées en antipyrine $\left(50 \mathrm{\gamma} / \mathrm{cm}^{3}\right.$ par exemple).

En fait, la densité optique propre de ces solutions n'intervient pas dans le deuxième temps du dosage, après addition de nitrite.

L'antipyrine responsable de cette absorption a disparu et s'est transformée, sous l'action du nitrite, en nitroso-antipyrine (fig. 2). 


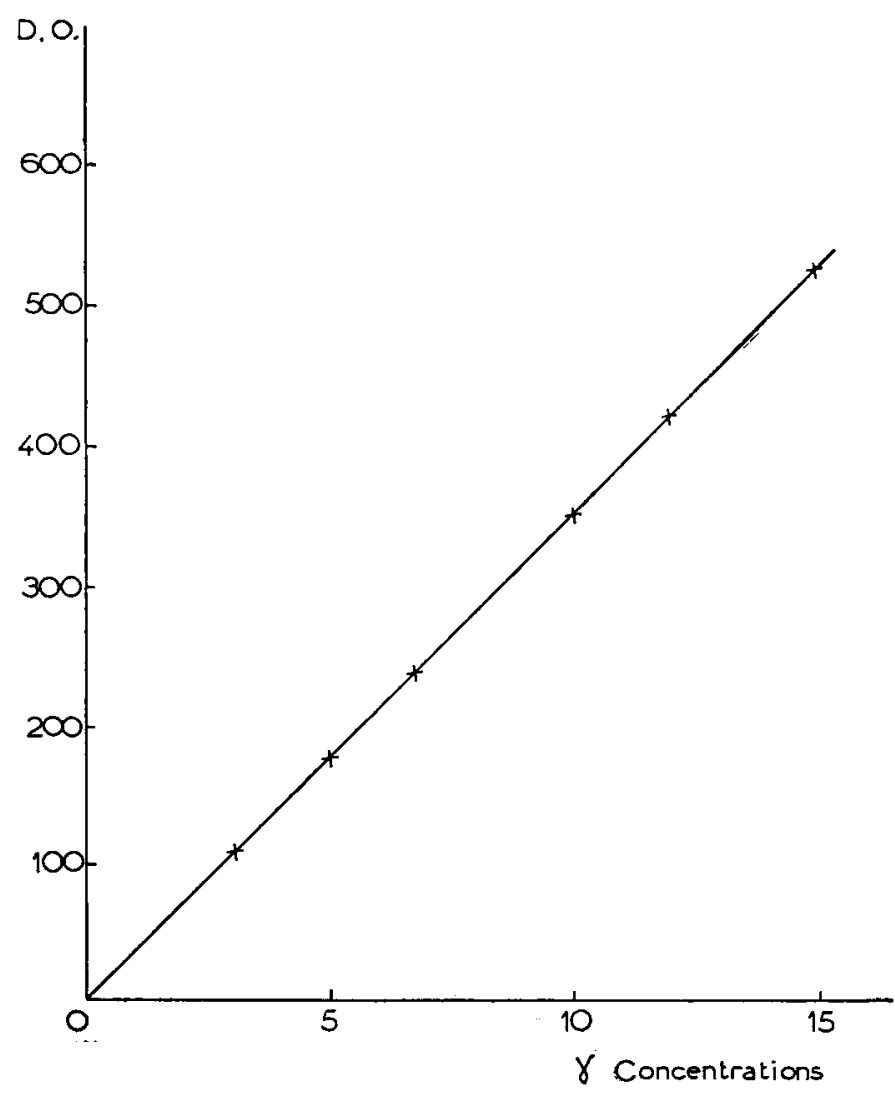

Fig. 2. - Densité optique en fonction de la concentration.

Les densités optiques des solutions de différentes concentrations ont été obtenues sans tenir compte de la densité propre de chaque solution par rapport à l'eau sulfurique. (Cette densité optique atteignait, pour certaines concentrations, I5 millièmes de densité optique.) Les densités optiques ainsi mesurées sont proportionnelles aux concentrations.

Les densités optiques portées en ordonnées des graphiques 2,4 à I 3 , sont les densités optiques des solutions par rapport à l'eau sulfurique.

\section{$2^{\circ}$ Cas des plasmas}

Rien ne vérifie expérimentalement 1'hypothèse de BrodIE, selon laquelle 1'absorption propre du milieu par rapport à l'eau sulfurique n'est pas modifiée par addition de nitrite et intervient intégralement dans l'absorption de la solution en milieu nitreux.

L'adjonction de nitrite entraîne, non seulement la formation d'un couple nitrite-acide sulfurique, mais également une réaction entre le nitrite et les constituants du plasma déféqué. Dans le cas des plasmas chargés en antipyrine, elle provoque, en outre, la formation de nitrosoantipyrine.

Dans le cas des sangs témoins (sangs prélevés avant l'injection d'antipyrine, donc sans antipyrine), la réaction entre le nitrite et les constituants du plasma déféqué entraîne une augmentation de la densité 
optique, variable d'ailleurs, mais toujours supérieure à la valeur de l'absorption de ce plasma par rapport à l'eau sulfurique.

Lors du dosage de l'antipyrine dans le plasma, la méthode à suivre devrait être la suivante :

Mesurer la densité optique du sang témoin prélevé arant l'injection d'antipyrine à l'animal par rapport au témoin nitrite $\left(^{1}\right)\left(3 \mathrm{~cm}^{3}\right.$ d'eau + I goutte de $\mathrm{SO}_{4} \mathrm{H}_{24} \mathrm{~N}+2$ gouttes de $\mathrm{NO}_{2} \mathrm{Na}$ à $\mathrm{o}, 2 \mathrm{p}$. IOo), soit $\mathrm{T}$.

Mesurer la densité optique d'un sang renfermant de l'antipyrine par rapport au témoin nitrite, soit $P$.

$\mathrm{P}$ - T représente l'absorption propre de la nitroso-antipyrine par rapport à l'eau sulfurique (fig. 3). L,es mesures effectuées de la sorte sur des D.O

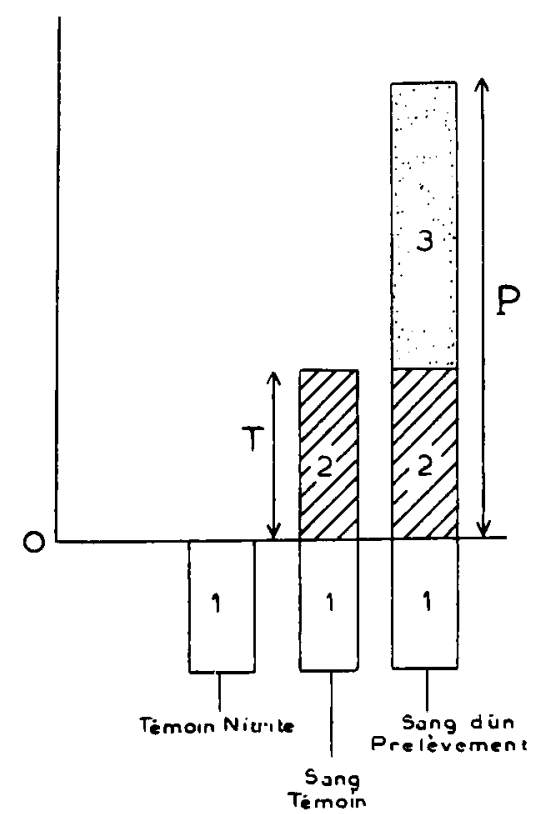

Fig. 3. - Io Absorption propre du témoin nitrite. -- $2^{\circ}$ Absorption de base du sang. $3^{0}$ Absorption due à la nitroso-antiprrine.

\section{TABLEAU I}

Exemple de surcharge de plasma de porc en antipyrine

\begin{tabular}{|c|c|c|}
\hline $\begin{array}{c}\text { Dilution } \\
\gamma \text { antipyrine/cc plasma }\end{array}$ & $\begin{array}{c}\text { Antipyrine retrouvé } \\
\text { Yoc plasma }\end{array}$ & $\%$ de recouvrement \\
\hline I25 $\% / 10,5 \mathrm{cc}$ & I I, 88 & 99,8 \\
\hline $250 \% / 10,5 \mathrm{cc}$ & 23,80 & 100 \\
\hline $375 \% / 1 \mathrm{I} \quad \mathrm{cc}$ & 34,08 & 99,0 \\
\hline $500 \% / 1 \mathrm{I} \quad \mathrm{cc}$ & +5.48 & 100,1 \\
\hline
\end{tabular}

(1) Il serait préférable, si l'on dispose d'un nombre suffisant de cuves, de conserver comme témoin le milieu sulfurique, car l'absorption du couple nitrite-acide sulfurique varie légèrement dans le temps et oblige à refaire constamment le zéro de l'appareil. 
sangs et des plasmas surchargés de quantités connues d'antipyrine confirment cette technique (tableau I). Les spectres d'absorption de la nitrosoantipyrine du sang obtenu dans ces conditions se superposent exactement à celui du produit pur (fig. 4).

D. 0

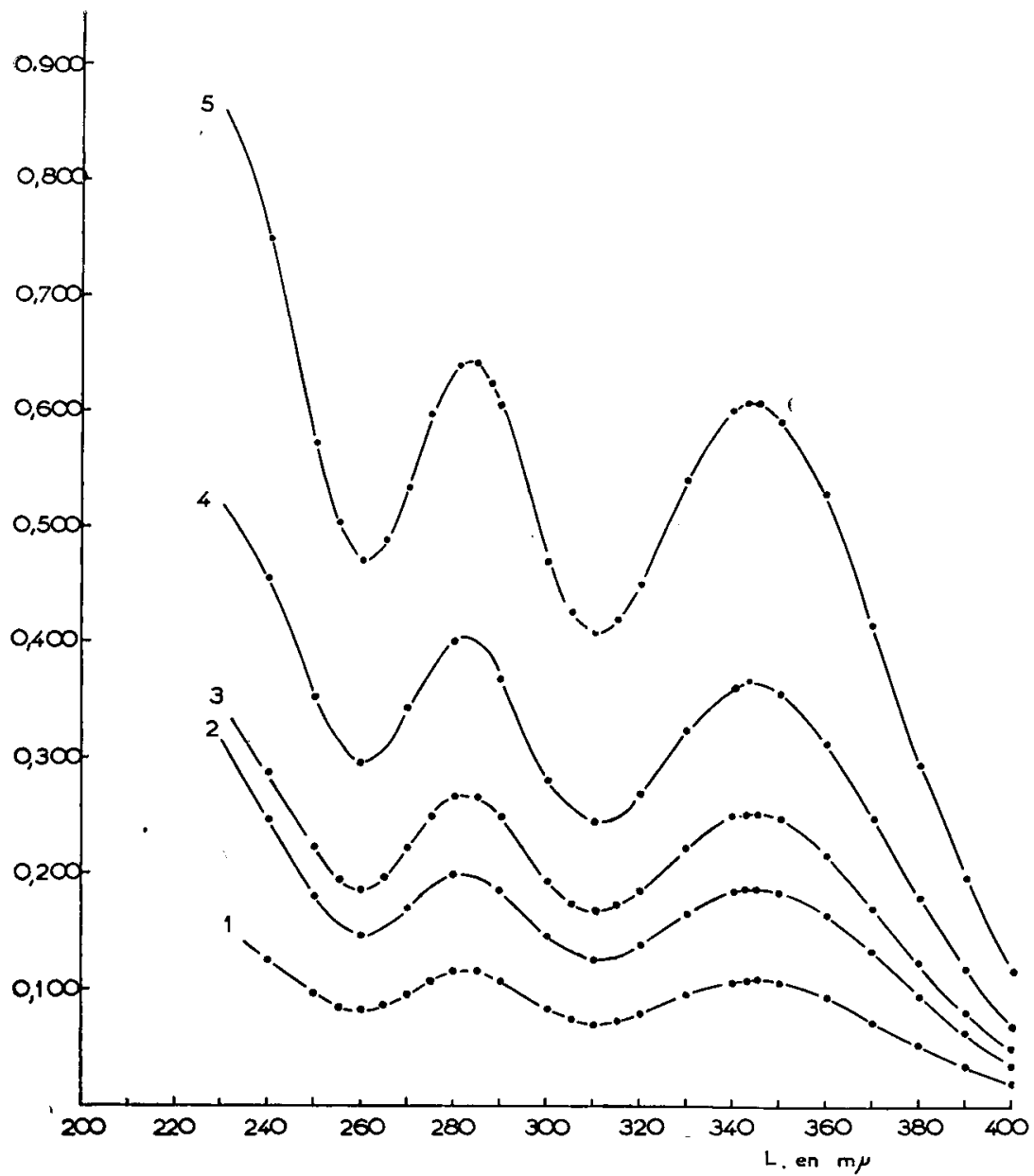

Figs. 4. - Variation de la densité optique de solutions d'antipyrine de différentes concentrations, en fonction de la longueur d'onde :

Courbes : I. $3 \% / \mathrm{cm}^{3}$ 2. $5 \% / \mathrm{cm}^{3} \quad 3.7 \% / \mathrm{cm}^{3} \quad+10 \% / \mathrm{cm}^{3} \quad 5 \cdot 15 \% / \mathrm{cm}^{3}$

\section{b) Longueur d'onde du dosage}

Le spectre de la nitroso-antipyrine/eau présente deux clochers: l'un à $343 \mathrm{mu}$;

l'autre à $283 \mathrm{~m} \mu$.

A ces deux longueurs d'onde, la densité optique des solutions est 


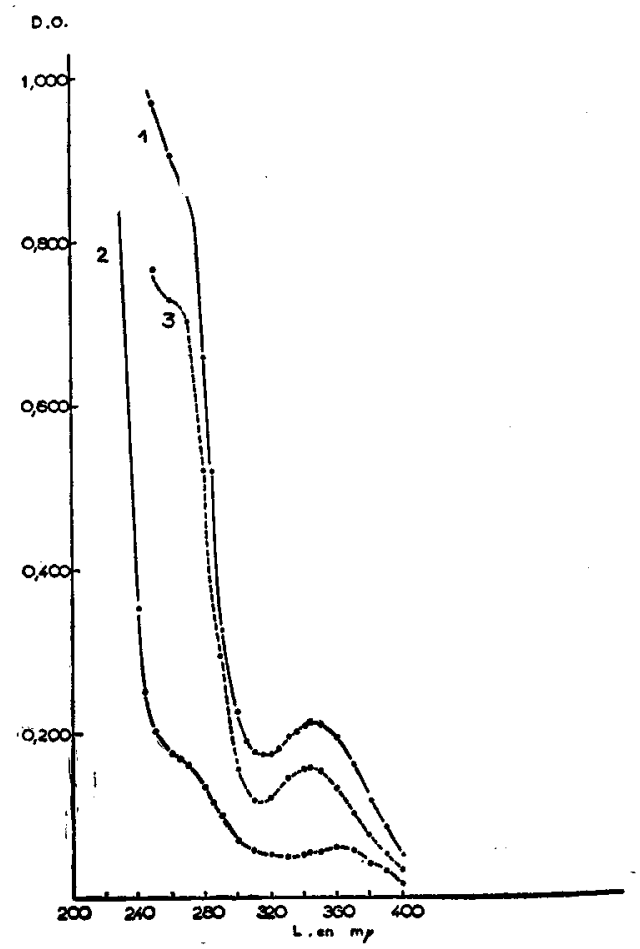

FIG.

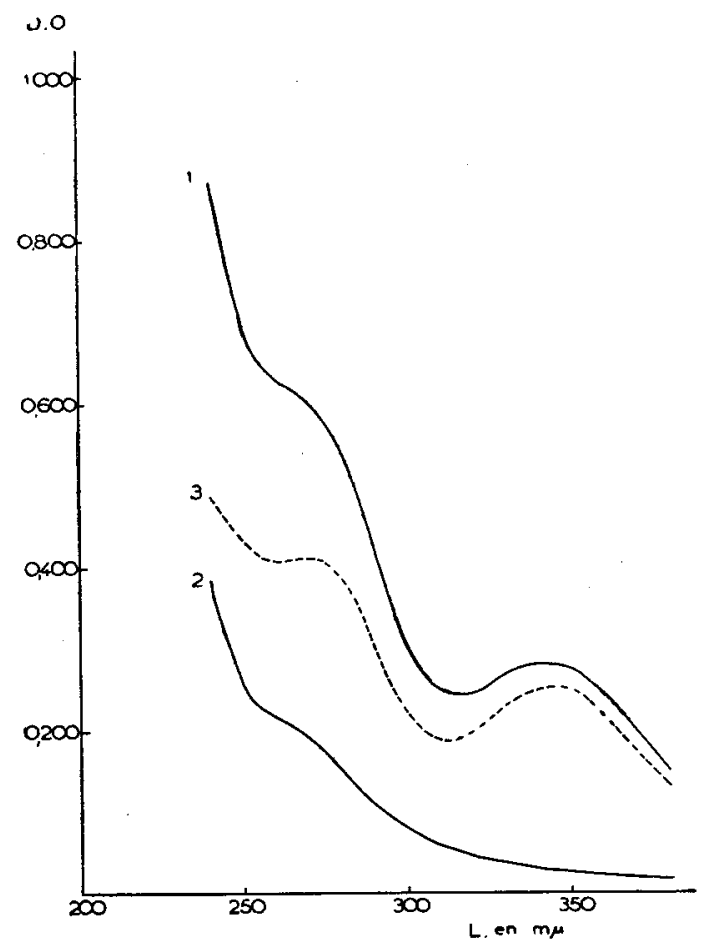

FIG. 6. 
proportionnelle à leur concentration en antipyrine. La longueur d'onde de $283 \mathrm{~m} u$ présentant l'absorption la plus élevée devrait être logiquement retenue. Il n'est toutefois pas possible de le faire, car la nitroso-antipyrine telle qu'on peut l'apprécier dans le sang d'animaux ayant reçu de l'antipyrine, ne présente pas de maximum d'absorption dans cette zone (fig. 5-6-7).

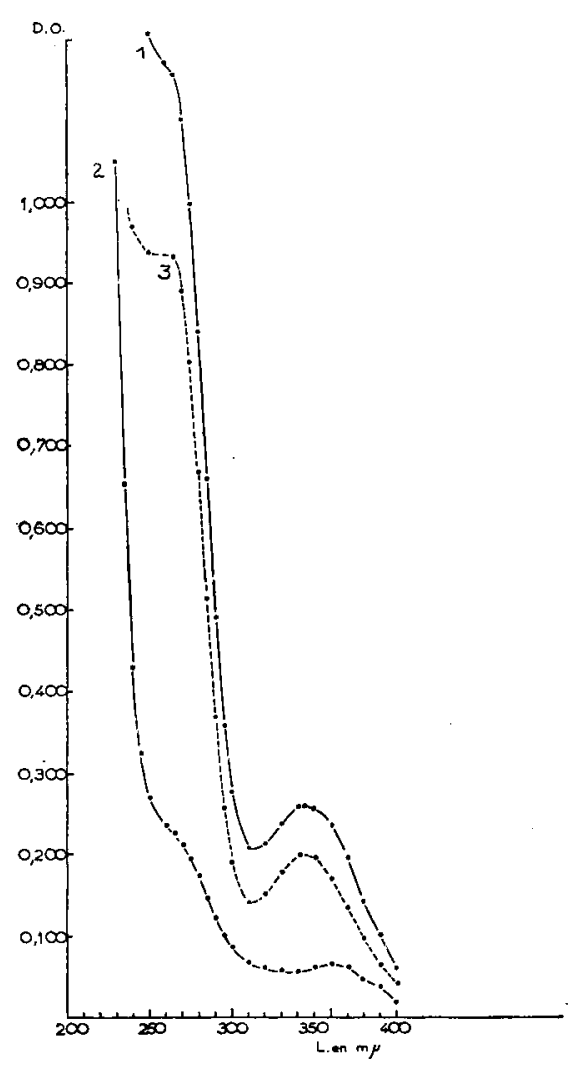

FIG,, .

Fig. $5-6--$

Variation, en fonction de la longrueur "l'onde, de la nitroso-antipyrine du sang le porcs ayant reçu de l'antiurine par voie veineuse :

I $^{\circ}$ Absorption totale du sang.

$2^{\circ}$ Absorption du sang témoin.

$3^{\circ}$ Absorption de la nitroso-antipyrine du sang.

Il est à noter, par contre, que si l'on surcharge artificiellement un sang ou un plasma, on retrouve les deux clochers (fig. 8-9). Tout se passe comme si l'injection d'antipyrine à un animal entraînait la formation dans son sang d'une substance absorbant fortement dans la zone de $270 \mathrm{~m} \mu$. Ceci se retrouve aussi bien chez le porc que chez le mouton. Les variations observées dans la zone de 270 mu semblent dépendre de la dose d'anti- 


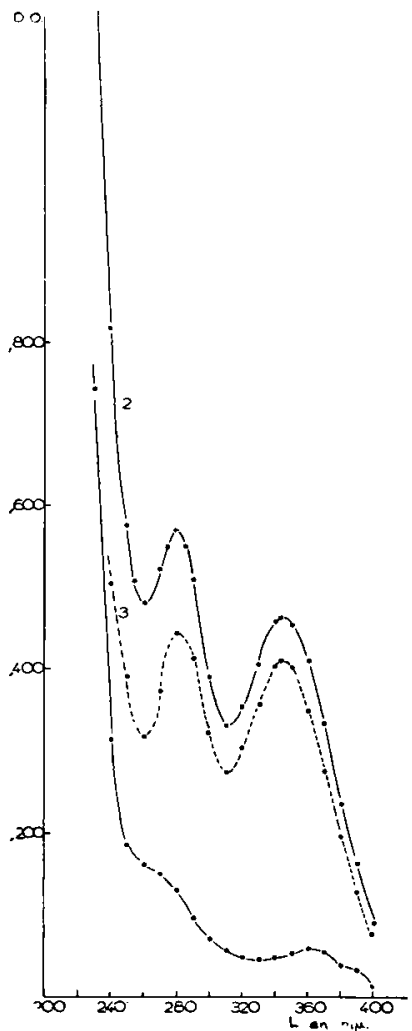

FIG. 8 .

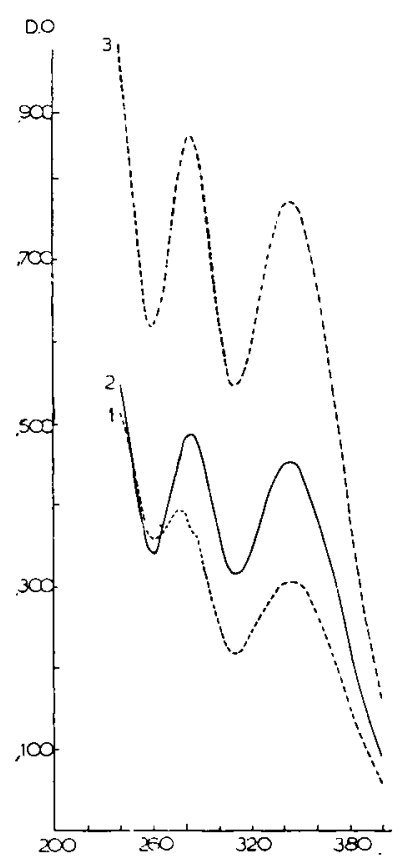

FIG. 9.

Fig. 8. - Variation de la densité optique de la nitroso-antipyrine d'un sang de porc surchargé artificiellement en antipyrine :

$\mathrm{I}^{\circ}$ Absorption du sang témoin sans antipyrine.

$2^{\circ}$ Absorption totale du sang surchargé.

$3^{\circ}$ Absorption de la nitroso-antipyrine du sang surchargé.

Fig. 9. - Comparaisons de la variation de la densité optique de la nitroso-antipyrine du sang de mouton ayant reçu de l'antipyrine par voie veineuse (courbe I en pointillé) et celle du plasma (courbe 2) et du sang (courbe 3) surchargés artificiellement et obtenus sur le même animal, le même jour avant l'injection d'antipyrine.

pyrine injectée et du temps séparant le prélèvement considéré du moment de l'injection.

La longueur d'onde à utiliser pour le dosage est celle du second maximum d'absorption : $343 \mathrm{~m} \mu$, et non $350 \mathrm{~m} \mu$ comme l'indique BRODIE. Les différences d'absorption observées à ces deux longueurs d'onde augmentent très sensiblement, au fur et à mesure que la concentration des solutions augmente (fig. Io). Jusqư'à $I_{5} \gamma / \mathrm{cm}^{3}$ la différence est pratiquement proportionnelle à la concentration (fig. II), et le fait de travailler à $35^{\circ} \mathrm{m} \mu$ n'entraîne pas d'erreurs importantes. Par contre, à des concentrations plus élevées, à $350 \mathrm{~m} \mu$, la densité optique des solutions n'est plus proportionnelle à leurs concentrations. 

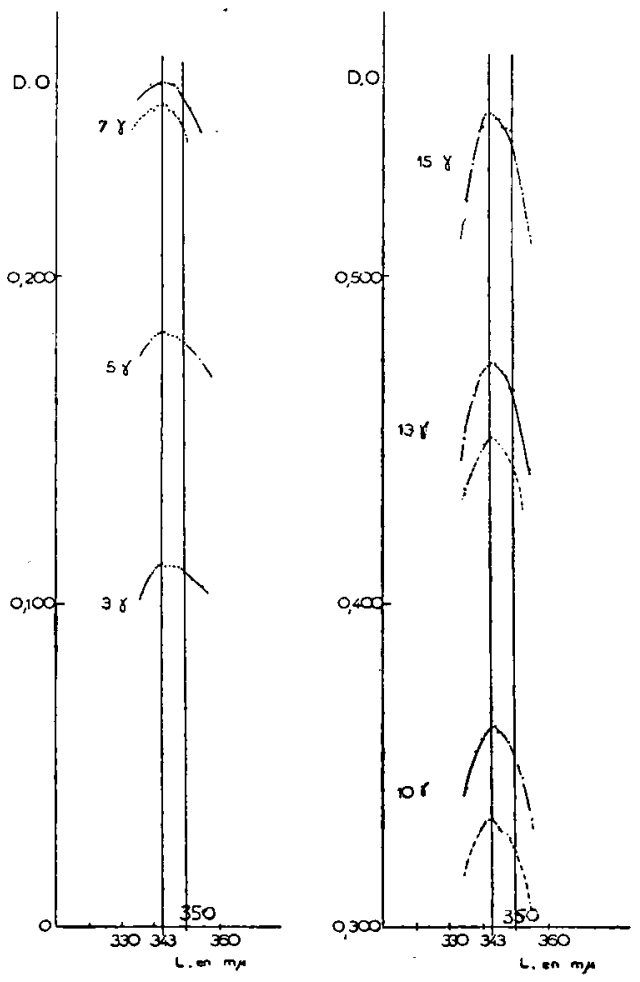

Fig. ro. - Sur les 2 lignes verticales sont représentées les valeurs des densités optiques de solutions de différentes concentrations d'antipyrine mesurées à 343 et $35^{\circ}$ mu. Au voisinage des courbes relatives à 7 , Io et $13 \gamma$ figure en pointillé le détail de l'absorption de la nitroso-antipyrine de 3 échantillons de sang.

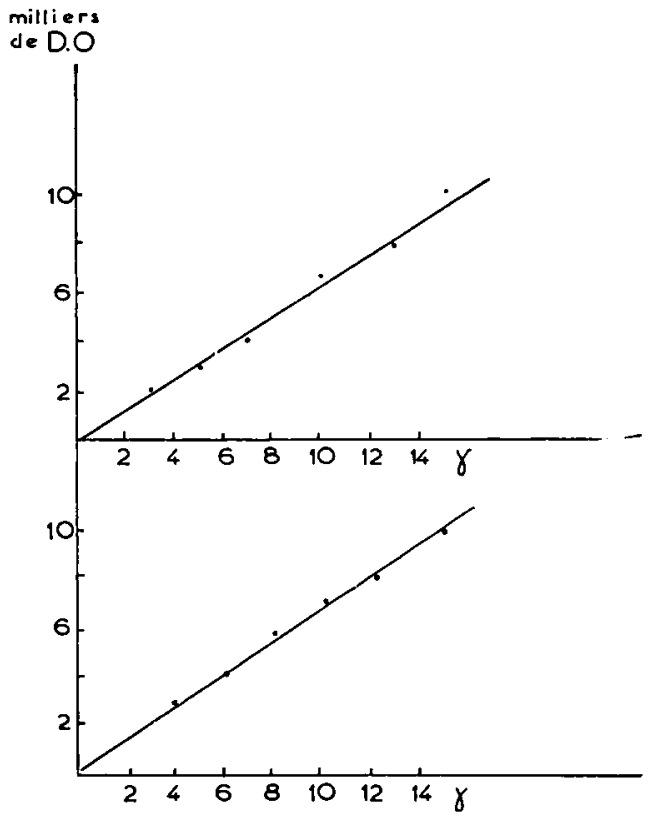

Fig. Ir. - Différences entre les valeurs de la densité optique pour diverses concentrations en antipyrine observée à 343 et $35^{\circ} \mathrm{m} \mu$ (les 2 courbes superposées se rapportent à 2 séries de mesure) 
Précisons qu'à $343 \mathrm{~m} \mu$, il est possible d'utiliser pour le dosage des cuves de Corex, au lieu de cuves en quartz.

\section{c) Conditions de lecture - Développement de la réaction}

La réaction de formation de la nitroso-antipyrine se développe assez lentement dans le temps et le maximum d'absorption est atteint à un temps plus ou moins long après l'addition de nitrite (fig. I2).

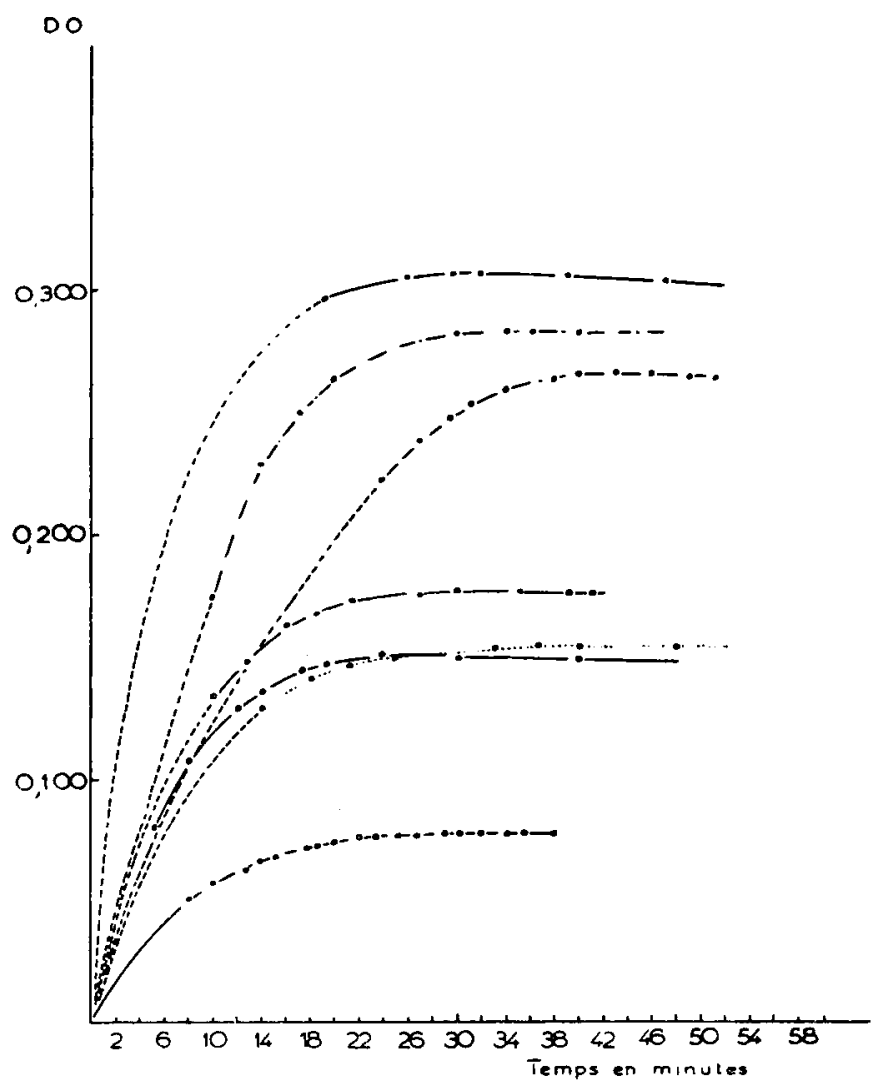

Fig. r 2. - Développement, en fonction du temps, de la formation de la nitrosoantipyrine de différents prélèvements de sang.

BRODIE indique de pratiquer la lecture 20 minutes après et précise que le maximum obtenu à ce moment se conserve environ jusqu'à 35 minutes, pour diminuer ensuite lentement.

En réalité, on ne peut donner de règles précises à ce sujet, car le temps de développement est très variable, non seulement pour des sangs, comme l'ont déjà signalé plusieurs auteurs (5-6), mais aussi pour le produit pur (témoin de ro $\gamma$ d'antipyrine par exemple) (tableau II). 
TABleau II

\begin{tabular}{|c|c|c|c|c|}
\hline & $\left|\begin{array}{l}\text { Concentration } \\
\text { antipyrine } / \mathrm{cc}\end{array}\right|$ & $\begin{array}{l}\text { Nombre } \\
\text { d'obser- } \\
\text { vations }\end{array}$ & Moyenne & Ecarts \\
\hline $\begin{array}{l}\text { Temps nécessaire pour obtenir } \\
\text { la valeur maximum } \ldots \ldots \\
\left.\text { Valeur maximum }{ }^{1}\right) \ldots \ldots\end{array}$ & $\begin{aligned} 7 & \ddots \\
\text { 10 } & \vdots \\
7 & \vdots \\
\text { I0 } & \vdots\end{aligned}$ & $\begin{array}{l}\text { I6 } \\
21 \\
\text { I6 } \\
40\end{array}$ & $\begin{array}{c}37 \mathrm{~m} 50 \mathrm{~s} \\
3+\mathrm{m} 30 \mathrm{~s} \\
252,6 \\
359,9\end{array}$ & $\begin{array}{r}25 \mathrm{~m}-57 \mathrm{~m} \\
23^{-}-48 \\
244-260 \\
340-373\end{array}$ \\
\hline
\end{tabular}

(1) En millièmes de densité optique.

La forme de la courbe du développement de la réaction en fonction du temps, bien que présentant toujours le même type général, est ellemême très variable (fig I3). Il importe donc de suivre avec précision le développement de la réaction par des lectures répétées, espacées de 2 en 2 minutes, à partir de 55 minutes après l'addition de nitrite, et de continuer les lectures un certain temps après avoir atteint la valeur la plus élevée. Il est possible d'enregistrer, au cours du dosage, de légères varia-

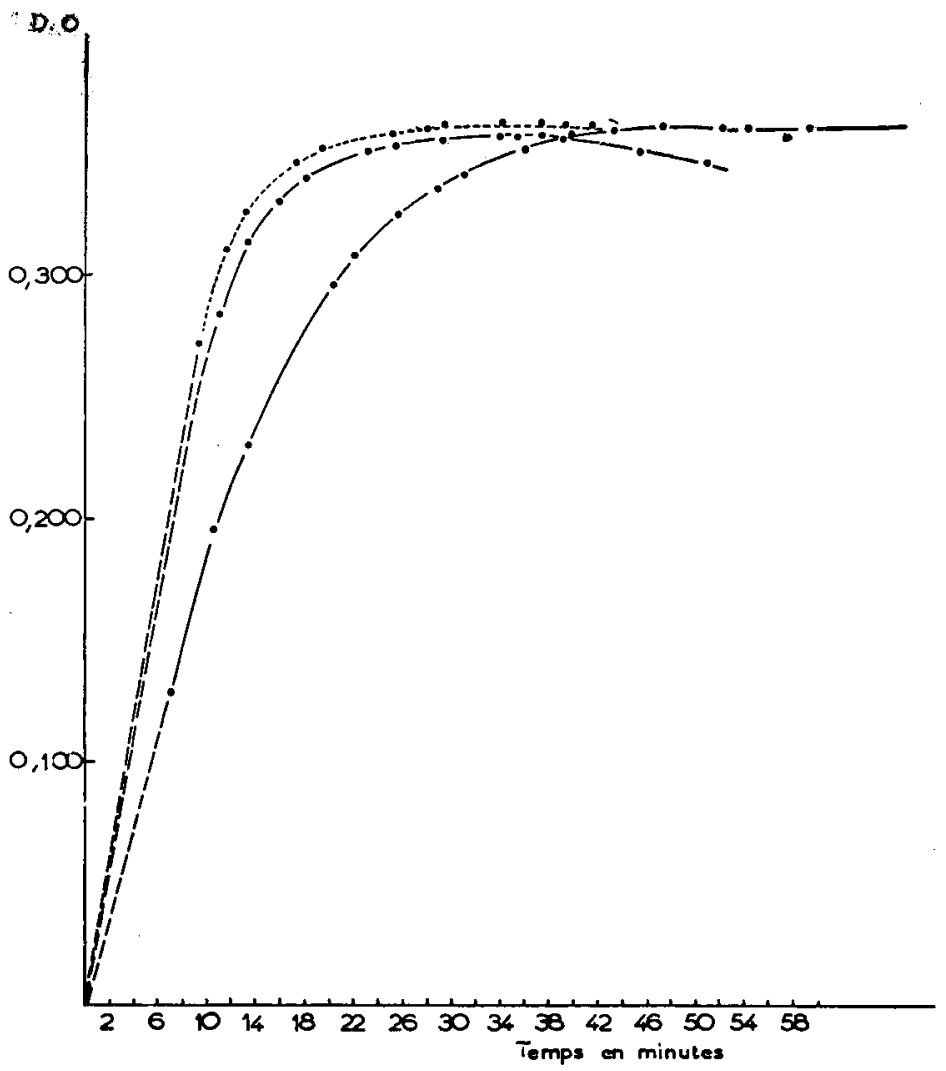

Fig. 13. - Développement, en fonction du temps, de la formation de nitroso-antipyrine à partir des solutions à $10 \% / \mathrm{cm}^{8}$. 
tions accidentelles. Il est recommandé de construire la courbe de développement de la réaction en fonction du temps, au fur et à mesure de son évolution au cours du dosage.

Chaque dosage doit être accompagné d'un dosage de produit pur (Io $\because$ d'antipyrine $/ \mathrm{cm}^{3}$ ). Son absorption moyenne est d'environ 360 et présente une variation importante d'une série de dosages à l'autre (tableau II).

Nous remercions M. Mocovor, Directeur de lat Station centrale de Microbiolorie et de Recherches Jaitieres, d'avoir nis a notre disposition son spectrophotometre BwckMan, pour effectuer tous les dosages nécessités par ce traval préliminaire de mise au point et par nos études sur la diffusion de l'antipyrine che' le porc et le mouton $(3-4)$.

Nous sommes très recommissants à M. François, Directeur du Service de Biochimie, d'avoir accepte de lire le manuscrit et de nous avoir fait part de ses observations.

\section{REFFRENCES BIBLIOGRAPHIQUES}

(I) Soberman (R.), Brodie (B. B.), Lévy (B.), Axelrod (J.), Hollander (V.), STEEI,E (J. M.). - J. Biol. Chem., 179, 3I-4I, I949.

(2) Brodie (B. B.), Axelrod (J.), Soblrman (R.), Lévy (B.). - J. Biol. Chem., 179, 25-30, 1949.

(3) Dumont (B. L.), Annales de Zootechnie IV, $x 955,305^{-3}$ I3.

(4) IJunow't (B. I.), Annales de Zootechnie IV, I955, 3I5-3I9.

(5) Scribante (P.), Maurice (P.), Favarger (P.). - Helv. Physiol. Acta, 10, 224-230, I952.

(6) Ikkos (D.), LUFT (R.), SJOGREN (B.). -- The J. of Clinical Investigation, 33, $7,989-94$, I 954 . 